\section{Generation of Cyanide Ion by the Reaction of Hexamine and Losartan Potassium with Sodium Hypochlorite}

\author{
Atsuko Adachi* and Toshio Okano \\ Department of Hygienic Sciences, Kobe Pharmaceutical Uni- \\ versity, 4 Motoyamakitamachi, Higashinada-ku, Kobe 658- \\ 8558, Japan
}

(Received April 30, 2008; Accepted July 2, 2008; Published online July 7, 2008)

The generation of cyanide ions by the reaction of pharmaceuticals containing nitrogen with sodium hypochlorite was examined using 20 different compounds. The generation of cyanide ions was observed in hexamine (hexamethylenetetramine), losartan potassium, metronidazole, and allopurinol. The largest generation was observed in hexamine. In both hexamine and losartan potassium, the amount of cyanide ion formed increased to a maximum at 45$60 \mathrm{~min}$. The generation of cyanide ions was attributed to the formation of nitroso product by oxidizing action of sodium hypochlorite.

Key words — cyanide ion, hexamine, losartan potassium, sodium hypochlorite

\section{INTRODUCTION}

Natural waters almost never contain detectable concentrations of cyanide ion, and so any amount found in water supplies is considered to be the result of man-made contamination. Even though the amount is small, the presence of cyanide ions in environmental water cannot be disregarded because of its high toxicity. ${ }^{1-3)}$

Pharmaceuticals are chemicals used for diagnosis, cure, mitigation or prevention of disease. Such drugs are used in large quantities in human. Many studies have now been conducted which show that drugs and their metabolites are widely distributed in surface waters. ${ }^{4-8)}$ The drugs detected

\footnotetext{
*To whom correspondence should be addressed: Department of Hygienic Sciences, Kobe Pharmaceutical University, 4 Motoyamakitamachi, Higashinada-ku, Kobe 658-8558, Japan. Tel.: +81-78-441-7525; Fax: +81-78-441-7565; E-mail: aadachi@kobepharma-u.ac.jp
}

in the environment were predominantly applied in human medicine. $\left.{ }^{6}\right)$ Free chlorine has been widely used as a disinfectant for urban wastewater treatment. ${ }^{9,10)}$ Treated water supplies may, in addition, contain species of higher oxidation states such as hypochlorous acid and hypochlorites, chloramines, and chlorine dioxide. ${ }^{11)}$ Sawamura et al. ${ }^{12)}$ demonstrated that hypochlorite reacts with amino acids to produce cyanide in aquatic environment. We predict that cyanide ion is generated by the reaction of pharmaceuticals containing nitrogen with sodium hypochlorite in water. Therefore, we tried to carry out fundamental research on the generation of cyanide ion in water on the laboratory scale.

No the generation studies of cyanide ion by the reaction of pharmaceuticals containing nitrogen with sodium hypochlorite in water have been reported. According to our knowledge, this is the first report on pharmaceuticals. The results are reported herein.

\section{MATERIAL AND METHODS}

Materials — Hexamine, acyclovir, allopurinol, bicalutamide, cimetidine, dantrolene sodium, droperidol, domperidone, dacarbazine, glimepiride, lansoprrazole, losartan potassium, lysozyme, miconazole nitrate, metronidazole, midazolam, naphazoline hydrochloride, phenytoin, pimozide and sodium hypochlorite solution of practical grade were purchased from Wako Pure Chemical Industries Ltd. (Osaka, Japan). Rice bran was purchased at a local market.

Procedure — An aliquot $(100 \mathrm{ml})$ of sample solution including pharmaceuticals $\left(0.5 \times 10^{-5}\right.$ $8.6 \times 10^{-5} \mathrm{M}$ ) was placed in a $200 \mathrm{ml}$ glass stoppered Erlenmeyer flask, to which sodium hypochlorite solution $\left(0.2 \times 10^{-4}-3.8 \times 10^{-4} \mathrm{M}\right)$ was added, and the solution was reacted at room temperature. After 15-120 min of reaction, rice bran $(1 \mathrm{~g})$ was added to stop the reaction. We have previously reported that sodium hypochlorite is decomposed rapidly by direct reaction with rice bran. ${ }^{13)}$ After $10 \mathrm{~min}$, the reaction mixture was filtered through filter paper to remove the rice bran. The concentration of cyanide ions in water samples was assayed by spectrophotometry using pyridinepyrazolone reagents after distillation according to the testing method for industrial waste water described in JIS (Japanese Industrial Standard) K 0102 (1986). ${ }^{14)}$ 
Table 1. Generation of Cyanide Ions from Pharmaceuticals Containing Nitrogen by Sodium Hypochlorite

\begin{tabular}{lcc}
\hline \hline Pharmaceuticals & $\begin{array}{c}\text { Concentration of } \\
\text { pharmaceuticals }(\mathrm{M})\end{array}$ & $\begin{array}{c}\text { Cyanide ion formed } \\
(\mathrm{mg} / \mathrm{l})\end{array}$ \\
\hline Hexamine & $6.5 \times 10^{-5}$ & 0.97 \\
Losartan potassium & $6.5 \times 10^{-5}$ & 0.65 \\
Allopurinol & $6.5 \times 10^{-5}$ & 0.02 \\
Metronidazole & $6.5 \times 10^{-5}$ & 0.01 \\
Dacarbazine & $6.5 \times 10^{-5}$ & - \\
Acyclovir & $6.5 \times 10^{-5}$ & - \\
Bicalutamide & $6.5 \times 10^{-5}$ & - \\
Cimetidine & $6.5 \times 10^{-5}$ & - \\
Clonidine hydrochloride & $6.5 \times 10^{-5}$ & - \\
Droperidol & $6.5 \times 10^{-5}$ & - \\
Domperidone & $6.5 \times 10^{-5}$ & - \\
Dacarbazine & $6.5 \times 10^{-5}$ & - \\
Glimepiride & $6.5 \times 10^{-5}$ & - \\
Lansoprrazole & $6.5 \times 10^{-5}$ & - \\
Lysozyme & $6.5 \times 10^{-5}$ & - \\
Miconazole nitrate & $6.5 \times 10^{-5}$ & - \\
Midazolam & $6.5 \times 10^{-5}$ & - \\
Naphazoline hydrochloride & $6.5 \times 10^{-5}$ & - \\
Phenytoin & $6.5 \times 10^{-5}$ & - \\
Pimozide & $6.5 \times 10^{-5}$ & - \\
\hline
\end{tabular}

\section{RESULTS AND DISCUSSION}

The generation of cyanide ions by the reaction of pharmaceuticals containing nitrogen with sodium hypochlorite was examined using 20 different compounds (Table 1). We chose these 20 compounds containing nitrogen from the handbook for new pharmaceuticals. ${ }^{15)}$ The generation of cyanide ions was observed in hexamine (hexamethylenetetramine), losartan potassium, metronidazole, dacarbazine and allopurinol.

The largest generation was observed in hexamine, followed by losartan potassium. From the above experiments, cyanide ion generation was further investigated with variation in reaction time and concentration of pharmaceuticals and sodium hypochlorite using hexamine and losartan potassium.

Figure 1 shows the time-courses of the generation of cyanide ion by the reaction of hexamine or losartan potassium with sodium hypochlorite. In hexamine, the amount of cyanide ion formed increased to a maximum at 45-60 min before decreasing. An increase of the reaction time from 75 to $120 \mathrm{~min}$ reduced the concentration from $0.8 \mathrm{mg} / \mathrm{l}$ to about $0.65 \mathrm{mg} / \mathrm{l}$. In losartan potassium, the amount of cyanide ion formed increased to a maximum at

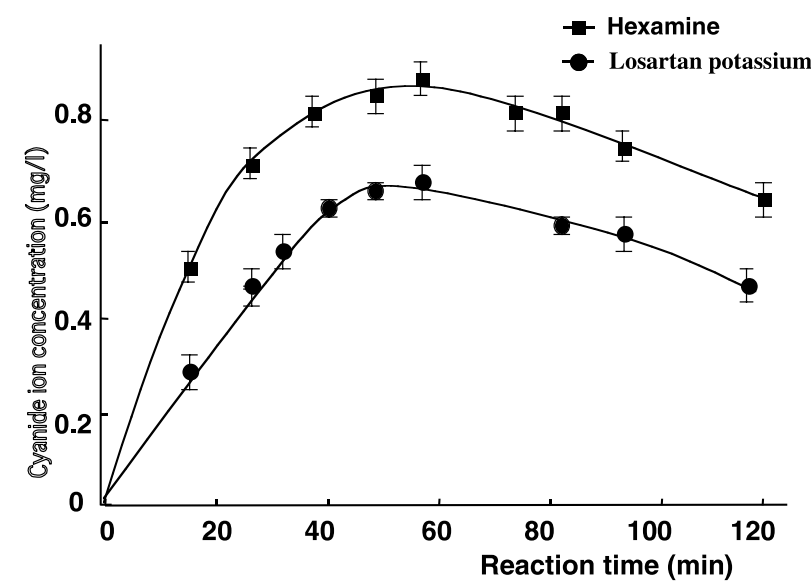

Fig. 1. Effect of Reaction Time on the Generation of Cyanide Ions by the Reaction of Hexamine or Losartan Potassium with Sodium Hypochlorite

Data represent the mean \pm S.D. of three separate experiments. Hexamine: $5.7 \times 10^{-5} \mathrm{M}$, Losartan potassium: $6.6 \times 10^{-5} \mathrm{M}$, Sodium hypochlorite: $1.34 \times 10^{-4} \mathrm{M}$.

$45-60 \mathrm{~min}$ as similar to that of hexamine. The effects of the amount of hexamine or losartan potassium on the generation of cyanide ion was investigated. As shown in Fig. 2, cyanide ion concentration increased in proportion to the amount of hexamine or losartan potassium.

Figure 3 shows the effect of the amount of sodium hypochlorite on the generation of cyanide 


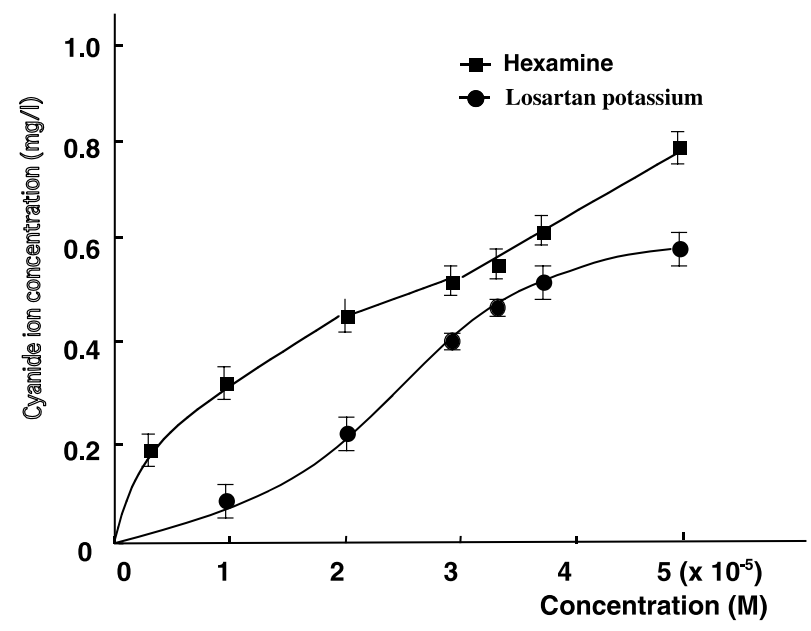

Fig. 2. Effect of Hexamine or Losartan Potassium on the Generation of Cyanide Ions

Data represent the mean \pm S.D. of three separate experiments. Sodium hypochlorite: $1.34 \times 10^{-4} \mathrm{M}$, Reaction time: $45 \mathrm{~min}$.

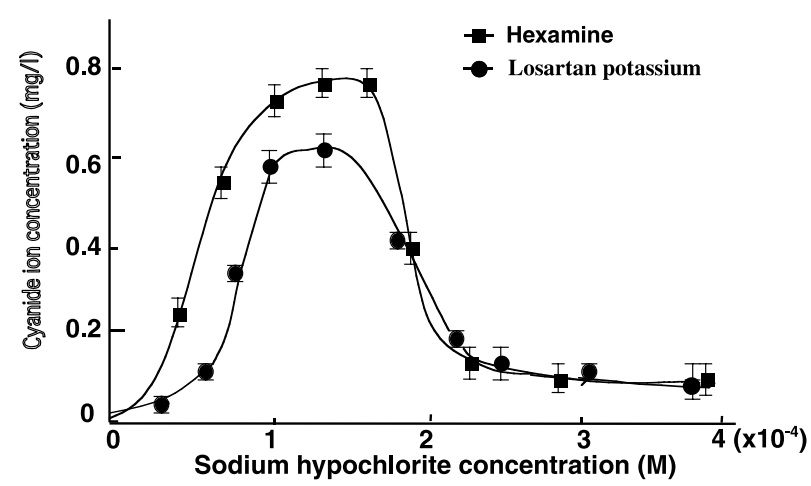

Fig. 3. Effect of Sodium Hypochlorite on the Generation of Cyanide Ions

Data represent the mean \pm S.D. of three separate experiments. Hexamine: $5.7 \times 10^{-5} \mathrm{M}$, Losartan potassium: $6.6 \times 10^{-5} \mathrm{M}$, Reaction time: $45 \mathrm{~min}$.

ion using hexamine or losartan potassium at a reaction time of $45 \mathrm{~min}$. The amount of cyanide ion formed increased to a maximum of $0.8 \mathrm{mg} / \mathrm{l}$ at the range of $1.3 \times 10^{-4}$ to $1.8 \times 10^{-4} \mathrm{M}$ of sodium hypochlorite and then decreased to $0.1 \mathrm{mg} / \mathrm{l}$ at $2.3 \times 10^{-4} \mathrm{M}$ of sodium hypochlorite. The decrease rate for concentrations above $1.8 \times 10^{-4} \mathrm{M}$ was very fast. In losartan potassium, the amount of cyanide ion formed increased to a maximum of $0.6 \mathrm{mg} / \mathrm{l}$ at the range of $1.0 \times 10^{-4}$ to $1.4 \times 10^{-4} \mathrm{M}$ of sodium hypochlorite and then reduced markedly to below $0.1 \mathrm{mg} / \mathrm{l}$.

The mechanism of the generation of cyanide ions was inferred as follows. The generation of cyanide ions was attributed to "Strecker degradation,"16) i.e., the formation of nitroso product by oxidizing action of sodium hypochlorite.

\section{REFERENCES}

1) Chen, K. K. and Rose, C. L. (1956) Treatment of acute cyanide poisoning. JAMA, 162, 1154-1157.

2) Wolfsie, J. H. and Shaffer, C. B. (1959) Hydrogen cyanide; hazards, toxicology, prevention and management of poisoning. J. Occup. Med., 1, 281-283.

3) Halstrom, F. and Moller, K. O. (1945) The content of cyanide in human organs from cases of poisoning with cyanide taken by mouth. Acta Pharmacol. Toxicol. (Copenh.), 1, 18-21.

4) Richardson, M. L. and Bowron, J. M. (1985) The fate of pharmaceutical chemicals in the aquatic environment. J. Pharm. Pharmacol., 37, 1-12.

5) Stumpf, M., Ternes, T. A., Wilken, R. D., Rodrigues, S. V. and Baumann, W. (1956) Polar drug residues in sewage and natural waters in the state of Rio de Janeiro, Brazil. Sci. Total Environ., 225, 135-141.

6) Ternes, T. A. (1998) Occurrence of drugs in German sewage treatment plants and rivers. Water Res., 32, 3245-3260.

7) Zuccato, E., Calamari, D., Natangelo, M. and Fanelli, R. (2000) Presence of therapeutic drugs in the environment. Lancet, 355, 1789-1790.

8) Reddersen, K., Heberer, T. H. and Dunnbier, U. (2002) Occurrence and identification of phenazone drugs and their metabolites in ground- and drinking water. Chemosphere, 49, 539-545.

9) Wolfe, R. L., Ward, N. R. and Olson, B. H. (1984) Inorganic chloramines as drinking water disinfectants. J. Am. Water Works Assoc., 76, 74-88.

10) Rook, J. J. (1976) Haloforms in drinking water. $J$. Am. Water Works Assoc., 68, 168-172.

11) Skinner, E. H. (1932) Results obtained from ammonia-chlorine treatment at Okmulgee. Water Works Engineering, 85, 1164-1168.

12) Sawamura, R., Sakurai, E., Yamamoto, M., Tachikawa, M. and Hasegawa, A. (1982) The reaction of hypochlorite with glycine. I. Decomposition of glycine and formation of cyanogens chloride. Eisei Kagaku, 28, 267-273 (in Japanese).

13) Adachi, A., Kimata, S., Noguchi, M. and Okano, T. (2002) Decomposition of monochloramione from water with rice bran. J. Health Sci., 48, 126-129.

14) Testing method for industrial waste water, (1986) JIS K 0102, p. 115.

15) Mizushima, U. (2008) Konnichinochiryouyaku in Japanese, In Handbook of new pharmaceuticals, Nankodo Press, Tokyo.

16) Schonberg, A. and Moubacher, R. (1952) The strecker degradation of $\alpha$-amino acids. Chem. Rev., 50, 261-277. 